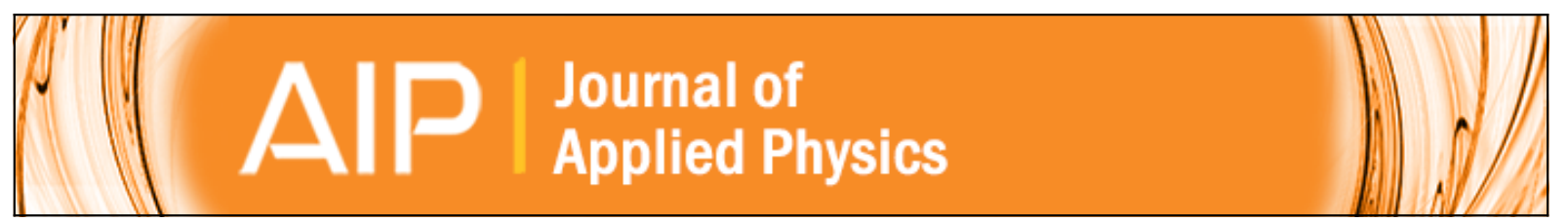

\title{
Enhancement in magnetic field sensitivity and reduction in equivalent magnetic noise by magnetoelectric laminate stacks
}

Menghui Li, Junqi Gao, Yaojin Wang, David Gray, Jiefang Li, and D. Viehland

Citation: Journal of Applied Physics 111, 104504 (2012); doi: 10.1063/1.4718441

View online: http://dx.doi.org/10.1063/1.4718441

View Table of Contents: http://scitation.aip.org/content/aip/journal/jap/111/10?ver=pdfcov

Published by the AIP Publishing

\section{Articles you may be interested in}

Influence of metglas layer on nonlinear magnetoelectric effect for magnetic field detection by frequency modulation

J. Appl. Phys. 117, 024104 (2015); 10.1063/1.4905622

Thermal stability of magnetoelectric sensors

Appl. Phys. Lett. 100, 173505 (2012); 10.1063/1.4705298

Dependence of magnetic field sensitivity of a magnetoelectric laminate sensor pair on separation distance: Effect of mutual inductance

J. Appl. Phys. 111, 033923 (2012); 10.1063/1.3684986

Enhanced dc magnetic field sensitivity by improved flux concentration in magnetoelectric laminates Appl. Phys. Lett. 99, 153502 (2011); 10.1063/1.3650713

Comparison of noise floor and sensitivity for different magnetoelectric laminates

J. Appl. Phys. 108, 084509 (2010); 10.1063/1.3486483

MIT LINCOLN

LABORATORY CAREERS

Discover the satisfaction of innovation and service

to the nation
- Space Control

- Air \& Missile Defense

- Communications Systems \& Cyber Security

- Intelligence, Surveillance and

Reconnaissance Systems

- Advanced
Electronics
- Tactical Systems
- Homeland
Protection
- Air Traffic Control

LINCOLN LABORATORY

MassachusetTs Institute of TeChNOLOGY

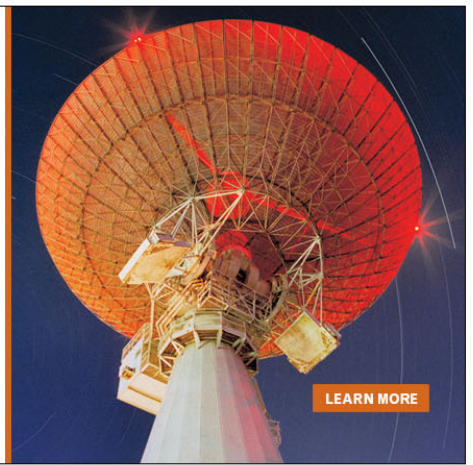




\title{
Enhancement in magnetic field sensitivity and reduction in equivalent magnetic noise by magnetoelectric laminate stacks
}

\author{
Menghui Li, ${ }^{a}$ Junqi Gao, Yaojin Wang, David Gray, Jiefang Li, and D. Viehland \\ Department of Materials Science and Engineering, Virginia Tech, Blacksburg, Virginia 24061, USA
}

(Received 2 March 2012; accepted 23 April 2012; published online 21 May 2012)

\begin{abstract}
We have investigated the equivalent magnetic noise and magnetic field sensitivity for a magnetoelectric (ME) sensor unit of $N$ numbers of ME laminates stacked together. Our results show with increasing $N$ that the modeled and measured equivalent magnetic noises decreased by a factor of $\sqrt{N}$ and that the magnetic field sensitivities increased by $\sqrt{N}$. For Metglas/ $\mathrm{Pb}\left(\mathrm{Mg}_{1 / 3}, \mathrm{Nb}_{2 / 3}\right)$ $\mathrm{O}_{3}-\mathrm{PbTiO}_{3}$ laminates, the equivalent magnetic noise decreased and the magnetic field sensitivity increased by a factors of 2.1 and 2.3 , respectively, for $N=4$ relative to that for $N=1$. () 2012 American Institute of Physics. [http://dx.doi.org/10.1063/1.4718441]
\end{abstract}

\section{INTRODUCTION}

The magnetoelectric (ME) effect-the induction of magnetization by an applied electric field (E), or a change in polarization by an applied magnetic field $(\mathrm{H})$ - - has been of recent research interests. ${ }^{1-4}$ Based on a giant ME effect, two phase piezoelectric/magnetostrictive laminates have shown the potential for low frequency $\left(\leq 10^{2} \mathrm{~Hz}\right)$, low cost, low power, and room temperature magnetic sensors. ${ }^{5-7}$ A ME magnetic sensor with a low equivalent magnetic noise and a $10 \mathrm{pT}$ magnetic field sensitivity have recently been reported. ${ }^{8}$

A ME sensor with a high signal-to-noise ratio (SNR) requires not only a high value of $\alpha_{\mathrm{ME}}$ so as to achieve high output signals but also requires a low equivalent magnetic noise in the absence of an incident field. ${ }^{9}$ The highest value of $\alpha_{\mathrm{ME}}$ reported for Metglas $/ \mathrm{Pb}(\mathrm{Zr}, \mathrm{Ti}) \mathrm{O}_{3}$ (PZT) two phase laminates is $22 \mathrm{~V} / \mathrm{cm} \cdot$ Oe. ${ }^{5,10}$ Much higher values of $\alpha_{\mathrm{ME}}=52 \mathrm{~V} / \mathrm{cm} \cdot$ Oe have been achieved for Metglas $/ \mathrm{Pb}$ $\left(\mathrm{Mg}_{1 / 3}, \mathrm{Nb}_{2 / 3}\right) \mathrm{O}_{3}-\mathrm{PbTiO}_{3}$ (PMN-PT) laminates. ${ }^{7}$ The reduction of the equivalent magnetic noise for ME magnetic sensors is difficult. It has been predicted that the stacking of $N$ numbers of ME laminates will increase the SNR by a factor of $\sqrt{N} \cdot{ }^{11,12}$ However, such theoretical and experimental studies of the effect of stacked arrays on the equivalent magnetic noise of Metglas/PMN-PT laminate sensors have not been reported.

Here, the equivalent magnetic noise has been reduced and the magnetic field sensitivity enhanced for Metglas/ PMN-PT laminates by stacking. Experimental results agree with predictions that the equivalent magnetic noise is decreased and magnetic field sensitivity increased both by factors of $\sqrt{N}$ with increasing $N$. ME sensor units of four laminates connected in parallel were found to have a $2.1 \times$ lower equivalent magnetic noise and a $2.3 \times$ higher magnetic field sensitivity.

\section{THEORY PREDICTION}

For ME sensors, the dominant internal noise sources have been identified to be the dielectric loss $\left(\mathrm{N}_{\mathrm{DE}}\right)$ and the

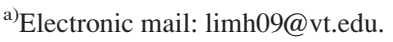

dc leakage resistance $\left(\mathrm{N}_{\mathrm{R}}\right)$ contributions. Secondary noise sources, such as thermal and electrical circuit noises, have been neglected due to a lower charge noise density relative to these internal sensor noise contributions. ${ }^{7}$ The charge noise density of these two dominant noise sources can be estimated as

$$
\begin{gathered}
N_{D E}=\sqrt{\frac{4 k T C \tan \delta}{2 \pi f}}, \\
N_{R}=\frac{1}{2 \pi f} \sqrt{\frac{4 k T}{R} .}
\end{gathered}
$$

The total charge noise density can then be given as

$$
N_{t}=\sqrt{N_{D E}^{2}+N_{R}^{2}}=\sqrt{\frac{4 k T C \tan \delta}{2 \pi f}+\frac{1}{(2 \pi f)^{2}} \frac{4 k T}{R}},
$$

where $k$ is Boltzmann's constant $\left(1.38 \times 10^{-23} \mathrm{~J} \mathrm{~K}^{-1}\right)$, T is the temperature in Kelvin, $\mathrm{C}$ is the capacitance, $\tan \delta$ is the dielectric loss, $\mathrm{R}$ is the dc resistance of the ME sensor, and $\mathrm{f}$ is the frequency in Hertz.

The equivalent magnetic noise $\mathrm{N}_{\mathrm{m}}$ can then be obtained from the $\alpha_{\mathrm{Q}}$ of the sensor and the total noise charge density

$$
N_{m}(\mathrm{~T} / \sqrt{H z})=\frac{N_{c}(\mathrm{C} / \sqrt{H z})}{\alpha_{Q}(\mathrm{C} / T)}=\frac{\sqrt{\frac{4 k T C \tan \delta}{2 \pi f}+\frac{1}{(2 \pi f)^{2}} \frac{4 k T}{R}}}{\alpha_{V} C} .
$$

If $N$ ME laminates are stacked in parallel, the capacitance of the array is $N$ times that of $\mathrm{C}$, the dc resistance is $1 / N$ and the dielectric loss is unchanged. Therefore, the equivalent magnetic noise of the ME array $N_{m A}$ can be estimated as

$$
N_{m A}=\frac{\sqrt{N\left(\frac{4 k T C \tan \delta}{2 \pi f}+\frac{1}{(2 \pi f)^{2}} \frac{4 k T}{R}\right)}}{N \alpha_{V} C}=\frac{1}{\sqrt{N}} N_{m} .
$$


From Eq. (5) and the predictions by Xing et al., ${ }^{11}$ one can see that the equivalent magnetic noise is decreased and the SNR is increased both by factors of $\sqrt{N}$.

\section{EXPERIMENTS}

We constructed several L-L multi-push-pull mode ME laminates and stacked them in a parallel mode. Two interdigitated Kapton electrodes were bonded to the top and bottom surfaces of a $40 \mathrm{~mm} \times 10 \mathrm{~mm}$ PMN-PT bundle, consisting of five $40 \mathrm{~mm} \times 2 \mathrm{~mm}$ PMN-PT fibers (Ceracomp, Korean) with epoxy resin (Stycast 1264, USA). Three $80 \mathrm{~mm} \times 10 \mathrm{~mm}$ Metglas foils (Vitrovac Inc., Hanau, Germany) were then laminated to both the top and bottom surfaces of the interdigitated electrode/PMN-PT core composite, with a different epoxy resins (West System 105/206, USA). More detailed information of the lamination process can be found in Ref. 10.

The value of $\alpha_{\mathrm{ME}}$ was measured using a lock-in amplifier (SR-850) in response to a pair of Helmholtz coils driven at an ac magnetic field of $\mathrm{H}_{\mathrm{ac}}=0.1 \mathrm{Oe}$ at a frequency of $f=1 \mathrm{kHz}$. Figure 1(a) shows the ME voltage coefficient $\alpha_{\mathrm{V}}$ and ME charge coefficient $\alpha_{\mathrm{Q}}$ as a function of $\mathrm{H}_{\mathrm{dc}}$ for a single Metglas/PMN-PT laminate. The maximum value of $\alpha_{\mathrm{v}}$ was $52 \mathrm{~V} / \mathrm{cm}$. Oe under a dc bias of $8.6 \mathrm{G}$; correspondingly, the maximum value of $\alpha_{\mathrm{Q}}$ was $2078 \mathrm{pC} / \mathrm{Oe}$. The ME coefficient reported here is significantly higher than that previ-
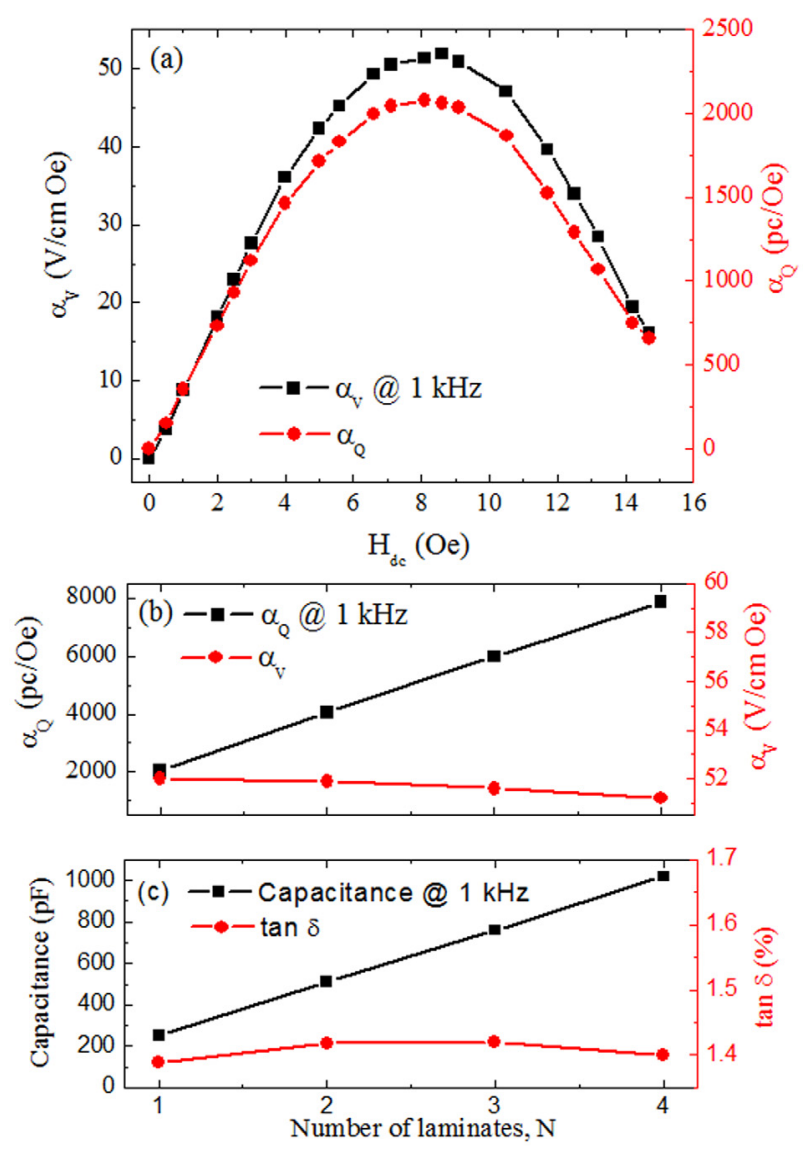

FIG. 1. ME voltage coefficient $\left(\alpha_{\mathrm{V}}\right)$ and ME charge coefficient $\left(\alpha_{\mathrm{Q}}\right)$ of Metglas/PMN-PT laminates as a function of (a) dc bias $\mathrm{H}_{\mathrm{dc}}$ at $1 \mathrm{kHz}$, and (b) the number of laminates stacked, $N$. (c) Capacitance and dielectric loss factor as a function of number laminates stacked, $N$. ously reported for two phase ME composites, which is mainly due to the high piezoelectric constant $\left(\mathrm{d}_{33, \mathrm{p}}\right)$ of the PMN-PT piezofibers. The maximum values of $\alpha_{\mathrm{v}}$ and $\alpha_{\mathrm{Q}}$ as a function of the number of laminates under the optimum $\mathrm{H}_{\mathrm{dc}}$ are shown in Fig. 1(b). The value of $\alpha_{\mathrm{v}}$ was unchanged and that of $\alpha_{\mathrm{Q}}$ increased linearly with $N$, agreeing well with predictions. Figure 1(c) shows the capacitance and the dielectric loss factor of the laminate array as a function of $N$. The capacitance increased linearly with $N$, and the dielectric loss factor was relatively invariant to $N$.

The laminates were then packaged with a simple lownoise charge amplifier having a gain of $5.1 \mathrm{~V} \mathrm{pC}^{-1}$ over the frequency range of $0.1<f<100 \mathrm{~Hz}$ (designed by SAIC), which we designate as a ME sensor unit. The unit was placed inside a high-mu-metal magnetic shielded chamber and connected to a dynamic signal analyzer to measure the voltage
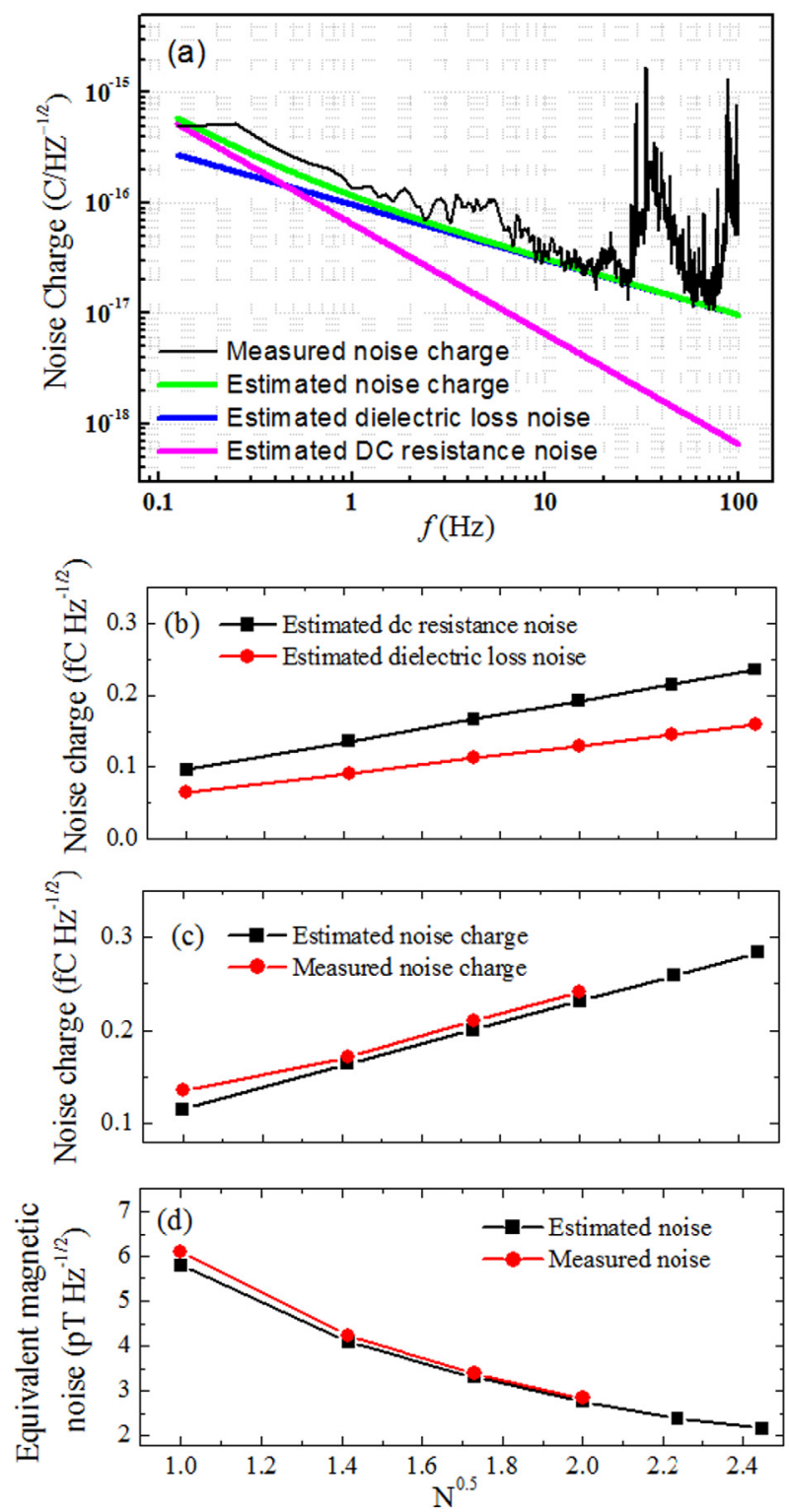

FIG. 2. (a) Measured and estimated charge noise density of the ME sensor unit for $\mathrm{N}=1$, including constituent dielectric loss and dc resistance, over the frequency range of $0.125<f<100 \mathrm{~Hz}$. (b) Estimated dc resistance and dielectric loss noise charge. Measured and estimated (c) noise charge and (d) equivalent magnetic noise at $1 \mathrm{~Hz}$, as a function of $\sqrt{N}$. 

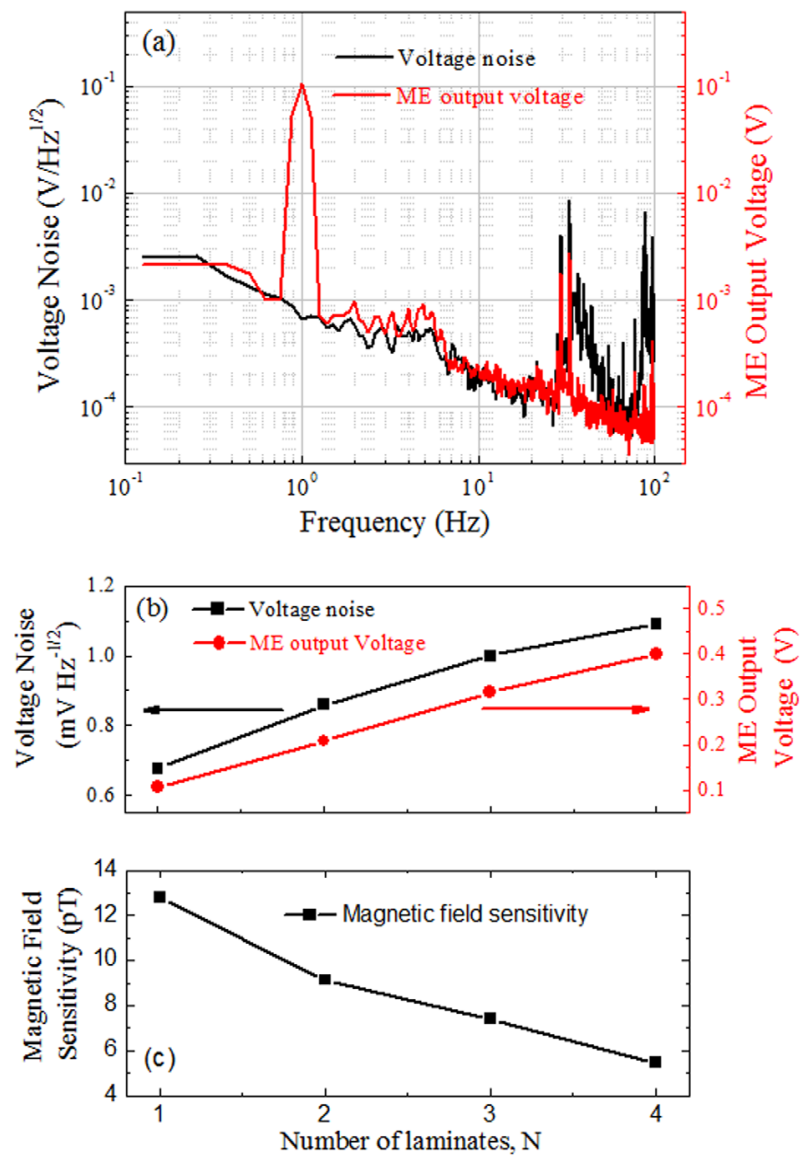

FIG. 3. (a) ME output signal of the ME sensor unit and background voltage noise in the absence of intentional excitation. (b) Voltage noise and ME output signal, and (c) magnetic field sensitivity of the sensor unit as a function of $\sqrt{N}$. The incident ac magnetic field was $1 \mathrm{nT}$ at $1 \mathrm{~Hz}$.

noise and output signal. Due to the dc resistance saturating the amplifier $(<30 \mathrm{G} \Omega)$, the largest number of laminates that we could connect in parallel and measure was $N=4$.

Figure 2(a) shows the measured and estimated charge noise spectra for a ME sensor unit which contained a single laminate. The noise density was estimated based on Eqs. (1)-(4), using appropriate ME laminate parameters. The estimated and measured charge noises exhibited good agreement, except at frequencies near external vibrational sources. Figures 2(b) and 2(c) show the estimated and measured charge noises for a ME sensor unit as a function of $N$ at $f=1 \mathrm{~Hz}$. In these figures, one can see that the estimated and measured noises increased proportionally to $\sqrt{N}$. Figure 2(d) shows the estimated and measured equivalent magnetic noises at $1 \mathrm{~Hz}$. The measured noise decreased with increasing $N$, which agreed well with the predicted noise. In particular, the equivalent magnetic noise of the ME sensor unit for $N=4$ was as low as $2.84 \mathrm{pT} / \mathrm{Hz}^{0.5}$, which was a factor of $2.1 \times$ lower than that for $N=1$. This low equivalent magnetic noise was also a factor of $1.8 \times$ lower than that previously reported for ME sensors. ${ }^{8}$ Such a low equivalent magnetic noise holds promise for ultra-low magnetic field detection applications at room temperature.
Next, the magnetic field sensitivity can be expressed as

$$
\text { Magnetic field sensitivity }=\frac{H_{a c-f}}{V_{M E-f}} \times S N R \times V_{\text {Noise }} \text {; }
$$

where $\mathrm{H}_{\mathrm{ac}-\mathrm{f}}$ is the ac magnetic field generated by a Helmholtz coil, $\mathrm{V}_{\mathrm{ME}-\mathrm{f}}$ is the ME output voltage of the ME sensor unit, $\mathrm{SNR}=2$, and $\mathrm{V}_{\text {Noise }}$ is the voltage noise at the frequency of interest. Figure 3(a) shows a voltage noise spectra and the output voltage of a $\mathrm{ME}$ sensor unit for $N=1$ over the frequency range of $0.125<f<100 \mathrm{~Hz}$, in response to a $1 \mathrm{nT}$ incident ac magnetic field at $1 \mathrm{~Hz}$. The output of the sensors was $0.106 \mathrm{~V}$, and the background voltage noise at $1 \mathrm{~Hz}$ was $6.77 \times 10^{-4} \mathrm{~V} / \mathrm{Hz}^{1 / 2}$. From Eq. (6), the magnetic field sensitivity for $N=1$ was determined to be $12.8 \mathrm{pT}$. In Figure 3(b), the voltage noise and output voltage of the ME sensor unit are shown as a function of $N$ in response to $\mathrm{H}_{\mathrm{ac}}=1 \mathrm{nT}$. The voltage noise and the output voltage increased with increasing $N$. Substituting the data of the voltage noise and the output voltage into Eq. (6), the magnetic field sensitivity was calculated as a function of $N$ as shown in Fig. 3(c). The magnetic field sensitivity for $N=4$ was 5.5 pT at $1 \mathrm{~Hz}$, which is a $2.3 \times$ enhancement relative to $N=1$. This increased sensitivity is a direct consequence of the increase in the ME charge coefficient that results from the ME laminate stacking.

\section{CONCLUSION}

In summary, the charge and equivalent magnetic noises of stacked ME laminate sensors have been investigated. Predicted and experimental data confirm that the equivalent magnetic noise decreased and the magnetic field sensitivity increased both by factors of $\sqrt{N}$. For $N=4$, the ME sensor had a $2.1 \times$ lower equivalent magnetic noise and a $2.3 \times$ higher magnetic field sensitivity than for a single laminate. The results demonstrate the potential of ME laminate arrays as low-noise, extremely sensitive magnetic field sensors.

${ }^{1}$ R. Ramesh and N. A. Spaldin, Nature Mater. 6, 21 (2007).

${ }^{2}$ N. A. Spaldin and M. Fiebig, Science 309, 391 (2005).

${ }^{3}$ C.-W. Nan, M. I. Bichurin, S. Dong, D. Viehland, and G. Srinivasan, J. Appl. Phys. 103, 031101 (2008).

${ }^{4}$ J. Ma, J. Hu, Z. Li, and C. W. Nan, Adv Mater. 23, 1062 (2011).

${ }^{5}$ S. Dong, J. Zhai, J. Li, and D. Viehland, Appl. Phys. Lett. 89, 252904 (2006).

${ }^{6}$ J. Zhai, Z. Xing, S. Dong, J. Li, and D. Viehland, Appl. Phys. Lett. 88, 062510 (2006).

${ }^{7}$ Z. Xing, J. Li, and D. Viehland, Appl. Phys. Lett. 91, 182902 (2007).

${ }^{8}$ Y. Wang, D. Gray, D. Berry, J. Gao, M. Li, J. Li, and D. Viehland, Adv Mater. 23, 4111 (2011).

${ }^{9}$ J. C. R. H. Koch, Science 242, 218 (1988)

${ }^{10}$ M. H. Li, D. Berry, J. Das, D. Gray, J. F. Li, and D. Viehland, J. Am. Ceram. Soc. 94, 3738 (2011).

${ }^{11}$ Z. Xing, J. Zhai, J. Gao, J. Li, and D. Viehland, IEEE Electron Device Lett. 30, 445 (2009).

${ }^{12}$ M. Li, Y. Wang, J. Gao, D. Gray, J. Li, and D. Viehland, J. Appl. Phys. 111, 033923 (2012). 